\title{
Does pain intensity predict a poor opioid response in cancer patients?
}

\author{
Sebastiano Mercadante ${ }^{a, d, *}$, Vittorio Gebbia ${ }^{b}$, Fabrizio David ${ }^{a}$, Federica Aielli ${ }^{e}$, \\ Lucilla Verna $^{e}$, Giampiero Porzio $^{e}$, Patrizia Ferrera ${ }^{a}$, Alessandra Casuccio ${ }^{c}$, \\ Corrado Ficorella ${ }^{e}$ \\ a Pain Relief and Palliative Care Unit, La Maddalena Cancer Center, Italy \\ b Department of Oncology, La Maddalena Cancer Center, Italy \\ ' Department of Oncology, University of L' Aquila, Italy \\ d Chair of Anesthesiology, Intensive Care and Emergency, and Palliative Medicine, University of Palermo, Italy \\ e L' Aquila per la vita, Home Care Service, Italy
}

\section{A R T I C L E I N F O}

Article history:

Received 20 October 2010

Received in revised form 11

December 2010

Accepted 15 December 2010

Available online 20 January 2011

Keywords:

Cancer pain

Assessment tools

Opioid response

\begin{abstract}
A B S T R A C T
Aim: The aim of this study was to test the hypothesis that initial pain intensity is not a predictive factor of poor opioid response in advanced cancer patients, as suggested by a recent work.

Methods: A secondary analysis of one-hundred-sixty-seven patients referred for treatment of cancer-related pain was conducted. Pain intensity at admission was recorded and patients were divided in three categories of pain intensity: mild, moderate and severe. Patients were offered a treatment with opioid dose titration, according to department policy. Data regarding opioid doses and pain intensity were collected after dose titration was completed. Four levels of opioid response were considered: (a) good pain control, with minimal opioid escalation and without relevant adverse effects; (b) good pain control requiring more aggressive opioid escalation, for example doubling the doses in four days; (c) adequate pain control associated with the occurrence of adverse effects; (d) poor pain control with adverse effects.

Results: Seventy-six, forty-four, forty-one and six patients showed a response a, b, c, and d, respectively. No correlation between baseline pain intensity categories and opioid response was found. Patients with response 'b' and 'd' showed higher values of OEI mg.

Conclusion: Baseline pain intensity does not predict the outcome after an appropriate opioid titration. It is likely that non-homogeneous pain treatment would have biased the outcome of a previous work.
\end{abstract}

(c) 2011 Elsevier Ltd. All rights reserved.

\section{Introduction}

The heterogeneity and complexity of patients with cancer pain have represented a relevant challenge for researchers in an attempt of grouping patients according to the character- istics of disease, pain and patients. ${ }^{1}$ The intention of a cancer pain classification has been to improve pain management and to predict the likelihood of successful pain treatment, possibly identifying cancer patients who are less likely to respond to standard treatment. The Edmonton Staging System

* Corresponding author: Address: Pain Relief and Palliative Care Unit, La Maddalena Cancer Center, Via San Lorenzo 312,90146 Palermo, Italy. Tel.: +39 0916806521; fax: +390916806110.

E-mail address: terapiadeldolore@lamaddalenanet.it (S. Mercadante).

0959-8049/\$ - see front matter @ 2011 Elsevier Ltd. All rights reserved.

doi:10.1016/j.ejca.2010.12.020 
(ESS) has been the first prognostic tool designed to enable researchers to speak a common language and to make meaningful generalisation from the results of clinical trials. ${ }^{2}$ Candidate variables to assess pain prognosis from different dimensions were analysed, and the prognostic factors were summarised in three prognostic stages. The accuracy of ESS was assessed in subsequent multicentre trials, as the original staging system demonstrated some weakness. In a new version of ESS (revised ESS and rESS), a different construct was hypothesised, according to which patients with less problematic pain features would require a shorter time to achieve stable pain control, require less complicated analgesic regimens, are more responsive to opioid therapy, and use lower opioid doses, ${ }^{3}$ accomplishing a more relative concept of opioid response. This classification was based on two stages, in which the original intermediate group was combined with the poor prognosis group. rESS had a better predictive value and a moderate to high inter-rater reliability. However, $60-67 \%$ of patients still achieved stable pain control, regardless of pain classification.

In a recent secondary analysis, other than previous factors examined including younger age, neuropathic pain, incident pain, psychological distress, baseline pain intensity have been found to have longer times to stable pain control, suggesting to incorporate this factor in a new staging system. ${ }^{1}$ This data were confirmed in an international multicentre validation study. Initial pain intensity was associated with more days to achieve stable pain control. ${ }^{4}$ High level of pain intensity, however, does not seem clinically an intrinsic factor, as may possibly depend on several factors, for example previous undertreatment, which per se could be resolved in a simple way and could not influence the prognosis. It could be argued that patients recruited in multicentre studies may receive different or less intensive treatment, which may influence the outcome.

The aim of this study was to clarify the role of pain intensity at admission as a predictive factor of poor pain control. To test this hypothesis data of advanced cancer patients assessed in a study of opioid response were reviewed in a secondary analysis. ${ }^{5}$ This group of patients were treated homogeneously, according to a clear protocol in acute palliative care units.

\section{Patients and methods}

This was a secondary analysis of a prospectively collected sample of one-hundred-sixty-seven patients referred for treatment of cancer-related pain to two acute pain relief and supportive care units during 18 month period. The original methods are described elsewhere. ${ }^{5}$ Before starting the treatment (T0) pain intensity in the past $24 \mathrm{~h}$ (average pain) was measured by using a numerical scale from 0 to 10 , choosing a number from 0 (no pain) to 10 (the worst pain imaginable). Patients were grouped in the ranges proposed by Fainsinger et al. ${ }^{1}$ : mild, 0-3; moderate, 4-6; and severe, 7-10. Patients were offered a treatment with opioid dose titration, balancing analgesia and adverse effects, eventually supported by symptomatic drugs, according to department policy and routine protocols in the units. The choice of opi- oids was individualised according to previous exposure and response, and tailored opioid titration was performed according to the pain intensity and patients' needs, eventually changing the route of administration, for example in patients with nausea and vomiting which precluded the oral route. An unfavourable opioid response, with a prevalence of adverse effects over analgesia despite supportive treatment, was followed by opioid switching, using starting conversion ratios previously described, ${ }^{6}$ with subsequent doses changed according to the clinical response. Data regarding opioid doses and symptom intensity were then repeated after dose titration was completed and opioid doses were stable or after a week (T1). Opioid dose stabilisation was considered as the planned daily dose requiring no more than two rescue doses as needed. Data were expressed in oral morphine equivalents.

At time of stabilisation (T1), the opioid response was clinically graded as follows: (a) good pain control (3 or less on a numerical scale 0-10) with minimal increases in opioid doses (less than doubling the doses) and without relevant adverse effects; (b) good pain control requiring more aggressive opioid escalation without adverse effects, for example doubling the doses in four days; (c) adequate pain control associated with the occurrence of adverse effects (intensity 2-3); (d) poor pain control with adverse effects. The opioid escalation index (OEI) was calculated as a percentage from the initial dose at T0-T1 (after a week), according to the following formula: OEI\%: $[(x-y) / y] /$ days $\times 100$, where $x$ is dose at stabilisation and $y$ is the dose at admission. OEI in $\mathrm{mg}$ was calculated with the following formula: $(x-y) /$ days, as oral morphine equivalents. This value has been used to express the need of opioid escalation in cancer patients. ${ }^{7}$

\subsection{Statistical analysis}

A power analysis indicate that a sample size of 25 patients per group would allow the detection of a $20 \%$ difference in pain intensity score $(P<0.05$, power $=0.8)$. This computation assumes that the mean difference is 0.20 with a $95 \%$ confidence interval of $0.07-0.33$ and the common within-group standard deviation of 0.28 .

Frequency analysis was performed with chi-square test. The chi-square for linear trend test was used to evaluate the different opioid response in the three pain intensity categories. A sample size of 30 patients for group was calculated to detect approximately a $20 \%$ effect size for individual items with $80 \%$ power at a significance level of $P<0.05$.

The paired Wilcoxon signed-rank test was used to compare pain intensity scores in the different treatment time. The one-way analysis of variance (ANOVA) and KruskalWallis test were used for parametric and non-parametric analysis, respectively, to evaluate differences between the groups. Spearman's rho correlation was used to test for a trend between PI and opioid response and opioid escalation indexes. All $P$ values were two-sided and $P$ values less than 0.05 were considered to indicate statistical significance. Data were analysed by the EpiInfo software (version 6.0, Centres for Disease Control and Prevention, Atlanta, GA, 
USA) and the SPSS Software (version 14.0, SPSS Inc., Chicago, Il, USA).

\section{Results}

The characteristics of patients and the number of patients with mild, moderate and severe pain at admission (and after titration) are described in Table 1 . One-hundred-three patients were males. The mean age was 65.7, 65.9 and 64.6 and mean Karnofsky status was 49.6, 52.5 and 55.2 in the three pain intensity categories, respectively. No differences in age, gender and Karnofsky status were found. The mean baseline pain intensity in the three categories of pain intensity was $2.3(1.1), 5.2(0.7)$ and $8.2(1.1)$, respectively. The mean pain intensity at stabilisation was 1.9 (1.2), 1.9 (1.3) and 2.8 (2.0), respectively. Opioid titration significantly decreased pain intensity in the three categories of baseline pain intensity, particularly in patients with severe pain (Table 1). The mean time to reach dose stabilisation (T1) was 2.8 days (range 1-6). No statistical differences between pain categories were observed (2.2, 3 and 3.4 days, respectively). The pain intensity at T1 was correlated to baseline pain intensity $(P=0.002)$.

Data regarding pain intensity categories, OEI and opioid response after opioid titration are reported in Tables 2 and 3. Only six patients had a response 'd' (poor pain control and adverse effects) and were not considered for eventual comparison. No differences in OEI in the three pain level categories were found.

The association between baseline pain intensity and opioid response was significant $(P=0.007)$. No statistical differences in the number of patients with each opioid response group were found amongst patients with different baseline pain intensities (linear trend test; Table 2).

No correlation between baseline pain intensity and opioid response (Spearman's rho correlation coefficient 0.023; 95\% $\mathrm{CI}=-0.129$ to $0.175, \mathrm{P}=0.765$ ) was found, as well as between baseline pain intensity groups and opioid response (Spearman's rho correlation coefficient $0.070 ; 95 \% \mathrm{CI}=-0.084$ to $0.220, P=0.373)$.

Data regarding the relationship between OEI and opioid responses are reported in Table 3. OEI was significantly associ- ated with response ' $b$ ' (good pain control requiring more aggressive opioid escalation), other than with response ' $d$ ' (poor pain control with adverse effects).

\section{Discussion}

Pain intensity at time of assessment has been recently indicated as a negative prognostic factor. Pain intensity was associated with a longer time to achieve stable pain control, high final opioid doses, and more complicated analgesic regimens. ${ }^{1}$ Our hypothesis was that pain intensity 'per se' cannot be considered to be predictive of a poor analgesic response, as it often depends on referral characteristics, for example treatment behaviour on behalf of previous teams or GPs, rather than on pain/patient characteristics. The reasons rely on the clinical experience of patients who are often undertreated at referral (and so present high pain intensity and receive inadequate therapy) and then respond to adequate changes of therapy, in some case just a timely increase in opioid doses. In this study, patients with different baseline pain intensities did not differ in opioid response after appropriate opioid titration.

The frequency comparison between baseline pain intensity and opioid response was significant $(P=0.007)$, probably because patients with moderate to severe pain were more likely to produce a response ' $b$ ' (that is patients having good pain control but requiring more aggressive opioid escalation). This result exactly reflects our expectation regarding patients who are undertreated and require an increase in opioid doses which, however, cannot be considered a problematic issue. However, different from Fainsinger's findings, this did not resulted in a more complex issue, giving that patients obtained adequate pain relief within about three days.

In another trial assessing pain prognostic factors, no factors were identified in a three weeks study, unless for worst pain severity after one week. Several surveys suggest that one week is adequate to assess an analgesic treatment. Most patients achieved pain relief $>80 \%$ by week one, with a median pain relief of $40 \%$ after one day, doubling the dose of oral morphine equivalents. ${ }^{8,9}$ Paradoxically, patients with highest pain scores were more likely to perceive better pain relief. In another study of one week duration, no single factor able to predict treatment outcome was identified. ${ }^{10}$

\begin{tabular}{|c|c|c|c|c|}
\hline & $\begin{array}{l}\text { Mild pain } \\
n=28\end{array}$ & $\begin{array}{l}\text { Moderate pain } \\
\quad n=98\end{array}$ & $\begin{array}{c}\text { Severe pain } \\
n=42\end{array}$ & $P$ \\
\hline Age mean $( \pm S D)$ & $65.7(12.2)$ & $65.9(11.5)$ & $64.6(10.9)$ & $0.825^{*}$ \\
\hline Gender $(\mathrm{M} / \mathrm{F})$ & $21 / 7$ & $61 / 37$ & $21 / 21$ & $0.102^{\wedge}$ \\
\hline Karnofsky mean $( \pm S D)$ & $49.6(6.0)$ & $52.5(9.9)$ & $55.2(10.4)$ & $0.056^{*}$ \\
\hline \multicolumn{5}{|l|}{ Primary tumour } \\
\hline Genitourinary & 6 (21.4\%) & 26 (26.5\%) & 6 (14.3\%) & \\
\hline Gastrointestinal & $8(28.6 \%)$ & $22(22.4 \%)$ & $16(38.0 \%)$ & \\
\hline Breast & $4(14.4 \%)$ & $6(6.1 \%)$ & $7(16.7 \%)$ & \\
\hline Lung & $5(17.8 \%)$ & $15(15.4 \%)$ & $6(14.3 \%)$ & \\
\hline Other & $5(17.8 \%)$ & $29(29.6 \%)$ & $7(16.7 \%)$ & \\
\hline Baseline pain intensity mean $( \pm S D)$ & $2.3(1.1)$ & $5.2(0.7)$ & $8.2(1.1)$ & \\
\hline Pain intensity after titration mean $( \pm S D)$ & $1.9(1.2)$ & $1.9(1.3)$ & $2.8(2.0)$ & $0.002^{*}$ \\
\hline
\end{tabular}




\begin{tabular}{|c|c|c|c|c|}
\hline & Mild pain $n=28$ & Moderate pain $n=98$ & Severe pain $n=42$ & $P$ \\
\hline OEI\% mean $( \pm S D)$ & 131 (297) & $182(560)$ & $179(278)$ & $0.873^{\mathrm{a}}$ \\
\hline OEI mg mean $( \pm S D)$ & $6.2(16.1)$ & $10.3(20.9)$ & $10.7(19.1)$ & $0.582^{\mathrm{a}}$ \\
\hline Response 'a' $N=76$ & $18(64.2 \%)$ & $40(40.8 \%)$ & $18(42.8 \%)$ & $0.007^{b}$ \\
\hline Response 'b' $N=44$ & $1(3.6 \%)$ & $30(30.6 \%)$ & $13(30.9 \%)$ & \\
\hline Response 'c' $N=41$ & $8(28.6 \%)$ & $27(27.6 \%)$ & $6(14.3 \%)$ & \\
\hline Response 'd' $N=6$ & $1(3.6 \%)$ & $1(1.0 \%)$ & $4(9.5 \%)$ & \\
\hline$P^{c}$ & $0.346^{c}$ & $0.667^{c}$ & $0.745^{c}$ & \\
\hline \multicolumn{5}{|c|}{$\begin{array}{l}\text { Response (a) good pain control, with minimal opioid escalation and without relevant adverse effects; (b) good pain control requiring more } \\
\text { aggressive opioid escalation, for example doubling the doses in four days; (c) adequate pain control associated with the occurrence of adverse } \\
\text { effects; (d) poor pain control with adverse effects. } \\
\text { Kruskal-Wallis statistic. } \\
\text { Fisher exact test. } \\
\text { c }\end{array}$} \\
\hline
\end{tabular}

\begin{tabular}{|c|c|c|c|c|c|}
\hline & Response 'a' $N=76$ & Response 'b' $N=44$ & Response 'c' $N=41$ & Response 'd' $N=6$ & $P$ \\
\hline OEI\% mean $( \pm S D)$ & 144 (259) & 139 (228) & $264(834)$ & $203.8(399)$ & $0.378^{a}$ \\
\hline OEI mg mean $( \pm S D)$ & $6.4(13.5)$ & $15.3(22.2)$ & $8.2(24.6)$ & $21.4(22.4)$ & $<0.0005^{a}$ \\
\hline
\end{tabular}

According to present data, baseline pain intensity level cannot be considered as an inevitable negative factor in pain prognosis. One possible explanation for the different findings of this study in respect to Fainsinger's studies, ${ }^{1,4}$ would rely on the method of titration and consequently the median length of time to stable pain control, which is a reasonable method reported in many studies of cancer pain. For example, in these two studies, patients with mild pain achieved pain control in a median of four-five days with final doses of parenteral morphine equivalents of $12-25 \mathrm{mg} /$ day. Patients with moderate pain achieved stable pain control after a median of eight-ten days with final doses of 48-64 mg/day. Finally, patients with severe pain required a median of thirteentwenty-two days with dose of $68-72 \mathrm{mg}$. In this study, the mean time to achieve adequate pain control was 2.8 days, suggesting that a more timely opioid titration can produce a rapid pain control in an appropriate setting, annulling the potential influence of initial pain intensity. Similar protocols were used in the two units involved in the present study. We can only suppose that different settings could have used different procedures of opioid titration, or that no established protocol was planned in multicentre studies performed in different centres.

In patients admitted to our acute palliative care unit, the time to achieve opioid dose stabilisation was about two days, with a decrease of pain intensity from 7.1 to 2.3 (on a numerical scale $0-10$ ), and an OEI\% of $52 .{ }^{11}$ Similarly, when patients changed their daily equianalgesic doses of parenteral morphine from $133 \mathrm{mg}$ to $213 \mathrm{mg}$, pain relief was achieved in the majority of patients within a week. ${ }^{8}$ Other experiences reported adequate pain control within $24-48 \mathrm{~h}$, independently of the initial pain intensity. ${ }^{12,13}$

This study also confirmed the real meaning of OEI, in the context of the concept of opioid responsiveness introduced in early nineties. ${ }^{14}$ Of interest, the equivalent morphine doses found in the Fainsinger's studies ${ }^{1,4}$ were not particularly high to justify such a slow titration. The need for opioid dose escalation as a feature of a pain classification system for advanced cancer patients has been assessed in a secondary analysis of a subsample of 532 advanced cancer patients. There were no significant associations between OEI\% and age, neuropathic pain, incident pain, psychological distress, or addictive behaviour. ${ }^{15}$ This outcome was expected, as OEI alone is just a tool able to monitor the opioid requirements for a determined period, rather than being an outcome itself. The 'opioid escalation index' (OEI) is a dynamic tool useful to describe a trend in opioid response, where multiple factors can be identified. On the other hand such a factor, expressed in the previous version of ESS as 'tolerance', ${ }^{16}$ had been already removed from the staging system. As expected, in this study OEI was associated with a response ' $b$ ', that is good pain control requiring more aggressive opioid escalation, exactly reproducing the meaning of this tool, that is the therapeutic effort to achieve a meaningful outcome. Of course, in patients with the worse response ('d'), opioid doses were escalated unsuccessfully, as no positive outcome was achieved within the selected time of one week. Only six patients (3.5\%) were considered unresponsive (response ' $d$ ') after one week, confirming the appropriateness of the opioid treatment. On the other hand the low number of patients in this group cannot allow to draw any specific conclusion from a statistical point of view. Further studies with a large cohort of patients, randomly selected for a subgroup analysis, should provide new information on this issue.

This study may have some limitations. Firstly, it was a secondary analysis. However, data were analysed through a rigorous assessment of specific parameters in a context of a prospective study. For the same reasons, this study did not 
evaluate other independent predictors of poor outcomes such as incident pain, psychological distress and alcoholism or chemical coping. ${ }^{1-3}$ As the distribution of these risk factors amongst the different groups in unknown, these findings should be considered preliminary and more research needs to be done on this important subject. Another limitation may derive by a more specific response to opioids, rather than to analgesic regimens also including adjuvant drugs acting as co-analgesics, due to the original protocol. However, data presented in terms of time to achieve a response seem to be even more effective with opioid titration alone than those produced in the recent rESS versions, where pain intensity was examined as a predictive factor for poor pain relief and other analgesic regimens were allowed. Finally, different approaches to titration, referral bias, different outcome measures and the small number of 'non-responders' may hide some common aspects between apparently different results.

In conclusion, data from this study suggest that initial pain intensity does not predict the outcome, whether an appropriate opioid titration is provided. The meaning of baseline pain intensity, the previous opioid therapy, and treatment behaviour as predictive factors of an analgesic response should be tested in studies with appropriate designs and similar protocols in opioid treatment. The lack of a common, valid, and homogeneously applied method for assessing therapy results still remains a major problem in research and clinical practice.

\section{Conflict of interest statement}

None declared.

\section{R E F E R E N C E S}

1. Fainsinger R, Fairchild A, Nekolaichuk C, et al. Is pain intensity a predictor of the complexity of cancer pain management? J Clin Oncol 2009;27:585-90.

2. Bruera E, MacMillan $\mathrm{K}$, Hanson J, MacDonald R. The Edmonton staging system for cancer pain: preliminary report. Pain 1989;37:203-9.
3. Fainsinger R, Nekolaichuk C, Lawlor P, et al. A multicenter study of the revised Edmonton staging system for classifying cancer pain in advanced cancer patients. J Pain Symptom Manage 2005;29:224-37.

4. Fainsinger RL, Nekolaichuk C, Lawlor P, et al. An intenrational muticentre validation study of a pain classification system for cancer patients. Eur J Cancer 2010;46:2896-904.

5. Mercadante S, Gebbia V, David F, et al. Tools for identifying cancer pain of predominantly neuropathic origin and opioid responsiveness in cancer patients. J Pain 2009;10:594-600.

6. Mercadante S, Ferrera P, Villari P, Casuccio A. Rapid switching between transdermal fentanyl and methadone in cancer patients. J Clin Oncol 2005;23:5229-334.

7. Mercadante S, Fulfaro F, Casuccio A, Barresi L. Investigation of an opioid response categorization in advanced cancer patients. J Pain Symptom Manage 1999;18:347-52.

8. Chang V, Hwang SS, Kasimis B. Longitudinal documentation of cancer pain management outcomes: a pilot study at a VA medical center. J Pain Symptom Manage 2002;24:494-505.

9. Hwang S, Chang V, Fairclough D, Kasimis B. Development of a cancer pain prognostic scale. J Pain Symptom Manage 2002;24:366-78.

10. Stromgren A, Groenvold M, Petersen M, et al. Pain characteristics and treatment outcome for advanced cancer patients during the first week of specialized palliative care. J Pain Symptom Manage 2004;27:104-13.

11. Mercadante S, Villari P, Ferrera P, Casuccio A. Opioid-induced or pain relief-reduced symptoms in advanced cancer patients? Eur J Pain 2006;10:153-9.

12. Manfredi PL, Chandler S, Pigazzi A, Payne R. Outcome of cancer pain consultations. Cancer 2000;89:920-4.

13. Klepstad P, Kaasa S, Skauge M, Borchgrevink PC. Pain intensity and side effects during titration of morphine to cancer pain patients using a fixed schedule dose escalation. Acta Anaestesiol Scand 2002;44:656-64.

14. Portenoy RK, Foley KM, Inturrisi CE. The nature of opioid responsiveness and its implications for neuropathic pain: new hypotheses derived from studies of opioid infusions. Pain 1990;43:273-86.

15. Lowe SS, Nekolaichuk CL, Fainsinger RL, Lawlor PG. Should the rate of opioid dose escalation be included as a feature in a cancer pain classification system? J Pain Symptom Manage 2008;35:51-7.

16. Bruera E, Schoeller T, Wenk R, et al. A prospective multicenter assessment of the Edmonton staging system for cancer pain. J Pain Symptom Manage 1995;10:348-55. 